\title{
Schumann Resonance Frequencies Found Within Quantitative Electroencephalographic Activity: Implications for Earth-Brain Interactions
}

\author{
Michael A. Persinger \\ Laurentian University, Sudbury, P3E 2C6, Ontario, Canada \\ E-mail address: mpersinger@laurentian.ca
}

\begin{abstract}
Recent measurements of cerebral quantitative electroencephalographic power densities within the first three harmonics of the earth-ionosphere Schumann resonances and the same order of magnitude for both systems electric and magnetic (pT) fields suggest the possibility of direct intercalation or interaction. The phase modulations of the Schumann propagations and those associated with consciousness are very similar. Quantitative solutions from contemporary values for the physical parameters of the human brain and the earth-ionospheric resonances suggest that electromagnetic information maintained during the first $30 \mathrm{~min}$ of experience could be also represented within a property of the (Hilbert) space occupied by the ionospheric wave guide within the earth's magnetic field. Several astronomical phenomena, including gravitational waves and the neutral hydrogen line, display physical properties with magnitudes matching cerebral electromagnetic activity particularly during light sleep. The presence of Schumann frequencies within the human brain may have greater significance than hereto assumed for the human species.
\end{abstract}

Keywords: Schumann resonances; quantitative electroencephalographic activity; convergence solutions; brain-wave guide interactions; gravitational waves; extracerebral memory representations

\section{INTRODUCTION}

During abiogenesis and the production of amino acids from atmospheric gases through electrical discharges ("lightning") [1], the fundamental Schumann resonances were prevalent within the semistable boundaries between the earth's surface and the ionosphere [2]. The fundamental frequency (first harmonic) of $\sim 7.5 \mathrm{~Hz}$ reflects the ratio of the velocity of light $\left(3 \cdot 10^{8} \mathrm{~m} \cdot \mathrm{s}^{-1}\right)$ and the earth's circumference $\left(\sim 4 \cdot 10^{7} \mathrm{~m}\right)$. Subsequent harmonics are separated by intervals of $\sim 6 \mathrm{~Hz}$. Koenig and his colleagues [3] described remarkable similarities between the shapes and patterns of the electromagnetic transients generated within this cavity and those recorded from the human brain by electroencephalography (EEG).

Improvements of the algorithms and sensitivities for quantitative measurements of human cerebral electroencephalographic activity (QEEG) have revealed the presence of at least the first three harmonics of the Schumann resonances within human cerebral activity. The measured and calculated values of the electric and magnetic fields are comparable to natural Schumann values. Here the implications of this verification of recondite Schumann 
resonances when integrated cerebral activity is measured directly for the potential representation of information within this system are developed. The potential chemical and physical bases for this representation are explored.

\section{SCHUMANN RESONANCE CHARACTERISTICS}

There are multiple reviews of the physical characteristics of the cavity or Schumann resonances [4]. The most recent (2014) text is by Nickolaenko and Hayakawa [5]. The harmonics or modes of the Schumann resonance peak around $7.8 \mathrm{~Hz}, 14.1 \mathrm{~Hz}, 20.3 \mathrm{~Hz}, 26.4$ $\mathrm{Hz}$, and $32.5 \mathrm{~Hz}[5,6]$. The typical strength of the electric field component is in the order of $\mathrm{mV} \cdot \mathrm{m}^{-1}$ while the magnetic field component is $\sim 1 \mathrm{pT} \cdot \mathrm{Hz}^{-1 / 2}$.

Compared to the troughs of about $1 \mathrm{pT}$ the peak intensities at the first and second harmonics are $\sim 3 \mathrm{pT}$ which decline to $\sim 1 \mathrm{pT}$ around $20.3 \mathrm{~Hz}$ and $<0.5 \mathrm{pT}$ at higher harmonics. The fundamental frequency and intensity increase by $\sim .04$ to $.12 \mathrm{~Hz}$ and 0.11 to $0.41 \mathrm{pT}$, respectively [7] during strong proton events. The coefficients of this magnitude are slightly but significantly different along the $\mathrm{X}, \mathrm{Y}, \mathrm{Z}$ axes of propagation.

Although these values appear minute, their potential can be realized by the magnetic energy from these values, described by:

$$
\mathrm{J}=\mathrm{B}^{2}(2 \mu)^{-1} \mathrm{~m}^{3}
$$

where $B$ is the magnetic field strength, $\mu$ is magnetic permeability $\left(4 \pi \cdot 10^{-7} \mathrm{~N} \cdot \mathrm{A}^{-2}\right)$, and $\mathrm{m}^{3}$ is volume of the human cerebrum $\left(\sim 1.3 \cdot 10^{-3} \mathrm{~m}^{3}\right)$. The solution is within the range of $\sim 10^{-20} \mathrm{~J}$. This quantum unit of energy is associated with the effect of the $1.2 \cdot 10^{-1} \mathrm{~V}$ net change during an action potential of a neuron upon a unit charge $\left(1.6 \cdot 10^{-19} \mathrm{~A} \cdot \mathrm{s}\right)$. That the activity of one neuron can affect the state of the entire cortical manifold has been shown experimentally [8]. In other words, this single quantum of energy $\left(10^{-20} \mathrm{~J}\right)$ from the Schumann resonance, represented within the whole human cerebrum, would have the potential to alter the state of activity by which experience and the representation of experience (memory) are strongly correlated.

Most of the energy from the earth-ionosphere resonance fields is derived from the persistent discharge of lightning within primary areas over the earth's surface. On average there are about 40 to 100 lightning discharges per second globally. This is the same range as the major "40 to 100 "Hz" activity associated with complex processes associated with complex cognition. Because of the very small attenuation rates within the cavity, electromagnetic patterns are generated for megameters without appreciable loss of amplitude. In general the expansion and return of the "ringing" around the earth's circumference can occur between 4 and 5 times. The latency for the return of the circular wave is about $125 \mathrm{~ms}$ [5]. Long term change (over five years) in amplitude of the magnetic field component varied by $3 \mathrm{pT}$ over a range of only $1 \mathrm{deg} \mathrm{C}$ in underlying surface temperature. This was attributed to the greater water vapour transport and hence thunderstorm activity when surface temperature of the earth increases.

Nickolaenko and Hayakawa's [5] recent text concerning the Schumann resonances indicates there are monthly variations of the first mode (first harmonic) that range between 7.8 and 8.0 $\mathrm{Hz}$. The duration variation in frequency shift, with a peak around $15 \mathrm{hr}$ UT, has been attributed to either the meridian drift in global lightning activity or global alternation in the height of the ionosphere. Amplitudes of the Schumann resonances (which reflect variations in 
global thunderstorm activity) exhibit a yearly maximum during May and a minimum in October-November with intrinsic periodicities of 5, 10, and 20 days. Increases in the third and fourth harmonic amplitudes with a shift of about $1 \mathrm{~Hz}$ in the peak frequency occur before some seismic events. Phase shifts of about 20 degrees have been measured for the fundamental between $7 \mathrm{~Hz}$ and $8.5 \mathrm{~Hz}$.

Assuming $125 \mathrm{~ms}$ cycle completion for the circular waves the shift would be approaching 20 to $25 \mathrm{~ms}$. This is the same interval associated with the "refresh rate" of the recursive rostral-caudal electromagnetic waves over the human cerebral cortices that specifically define the waking and the dream state [9] as well as the time required to add one base to a DNA sequence [10]. Although the close convergence between the temporal parameters that define repetition in the re-entrant processes associated with consciousness and the stacking sequences of the genetic determinants within the cell could explain the long suspected interplay between cognition and body function (health), the shared durations with earth-ionosphere phase shifts emphasize their potential contribution to both processes. The possibility that some feature of space within the earth-ionosphere cavity could contain a complex electromagnetic template for at least some components of DNA sequences is a concept to explore. We have strongly considered that a physical equivalence of Hilbert space [11] would accommodate the technical requirements.

\section{SCHUMANN-BRAIN INTENSITIES}

Spectral analyses of the measurement of normal brain activity measured in $\mu \mathrm{V}^{2} \mathrm{~Hz}^{-1}$ with 19 channel Mistar equipment [12] reveals typical peaks in standardized spectral power of around $10 \mathrm{~Hz}$ along the rostral-caudal axis and 2 to $3 \mathrm{~Hz}$ across the hemispheres. The beat frequency, the difference between the medians of these two peaks is remarkably similar to the 7 to $8 \mathrm{~Hz}$ band associated with the Schumann fundamental frequency. Direct spectral analyses of the averaged global quantitative electroencephalographic activity sampled 250 times per sec for $16 \mathrm{~s}$ reveals conspicuous increases in power density within the range of the fundamental (first harmonic) as well as the second and third harmonic of the Schumann resonances. The peaks for these amplifications, as shown in Figure 1, occurred at $7.8 \mathrm{~Hz}, 14$ $\mathrm{Hz}$, and $20 \mathrm{~Hz}$.

The averaged potential difference as measured by QEEG is about $3 \mu \mathrm{V} \cdot \mathrm{Hz}^{-1}$. Applied across the distances of measurement the value is $\sim 30 \mu \mathrm{V} \cdot \mathrm{m}^{-1} \cdot \mathrm{Hz}^{-1}$, which occupies the range for the electric components of the Schumann fields. It may be relevant that, when adjusted by simple topological transformations, the physical structure of the relatively conductive skull, the intervening space of air and diminished tissue density (meninges) and the very conductive cerebral cortices, reveals similar properties to the earth-ionosphere wave guide through which the Schumann resonances are propagated. The magnetic field associated with this changing potential difference can be estimated by employing magnetic diffusivity and average resistivity. Magnetic diffusivity can be derived from:

$$
\eta=\left(\mu_{\mathrm{o}} \sigma\right)^{-1}
$$

where $\mu_{0}$ is magnetic susceptibility $\left(4 \pi \cdot 10^{-7} \mathrm{~N} \cdot \mathrm{A}^{-2}\right)$ and $\sigma=$ electrical conductivity [13]. Assuming the average resistivity of $2 \Omega \cdot \mathrm{m}\left(\sigma \sim 0.5 \mathrm{~S} \mathrm{~m}^{-1}\right)$ for extracellular fluid, and the average potential difference of $3 \mu \mathrm{V}$, the resulting estimate for the magnetic field strength is $\left(3 \cdot 10^{-6} \mathrm{~kg} \cdot \mathrm{m}^{2} \cdot \mathrm{A}^{-1} \mathrm{~s}^{-3}\right)$ divided by $1.58 \cdot 10^{6} \mathrm{~m}^{2} \cdot \mathrm{s}^{-1}$ or $\sim 1.9 \cdot 10^{-12} \mathrm{~T}$, that is about 2 picoTeslas $(\mathrm{pT})$. 


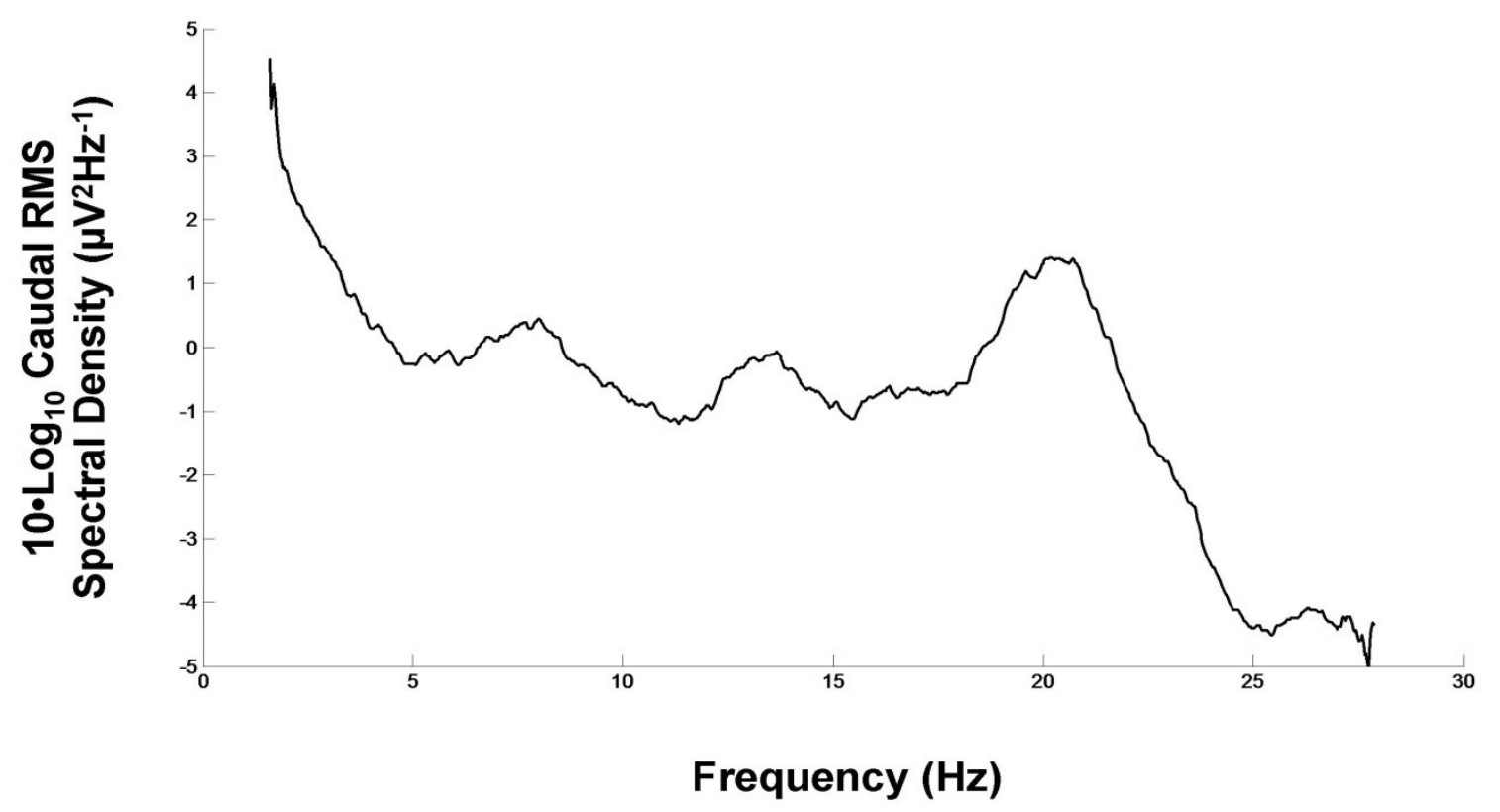

Figure 1. Calculated intensity based upon the cube root of the product of the spectral density of quantitative electroencephalographic activity over the caudal cerebral hemispheres $(\mathrm{T} 5, \mathrm{~T} 6, \mathrm{O} 1, \mathrm{O} 2, \mathrm{P} 3, \mathrm{P} 4, \mathrm{Pz})$ for 34 adult individuals. Note the elevation in power in the first three harmonics $(7-8 \mathrm{~Hz}, 13-14 \mathrm{~Hz}, 19-20 \mathrm{~Hz}$ ) of the Schumann resonances (from Kevin Saroka and Michael Persinger, unpublished data).

This intensity is within the range measured directly within a $\mathrm{cm}$ from frog sciatic nerve stimulation [14]. picoTesla strengths are also within the range of median values for intergalactic magnetic fields. The coefficient and order of magnitude are remarkably similar to those of the first three modes of the Schumann resonances.

\section{BRAIN SIGNIFICANCE OF SCHUMANN FREQUENCIES}

Nunez' [15] brilliant treatise entitled "Towards a physics of the neocortex" indicated that given the usual $4.5 \mathrm{~m} \cdot \mathrm{s}^{-1}$ bulk velocity of action potentials within the cerebral cortices, the approximate standing wave or resonance for a $60 \mathrm{~cm}$ circumference skull would be $\sim 7.5 \mathrm{~Hz}$. This bulk velocity would require $\sim 20$ to $25 \mathrm{~ms}$ to traverse the cerebral length and is equivalent to about 40 to $45 \mathrm{~Hz}$. The 40 to $45 \mathrm{~Hz}$ band is the classic frequency associated with "consciousness" and other complex biological functions. As demonstrated by Llinas and his colleagues [16] the " 40 " $\mathrm{Hz}$ repetition and phase modulation of an integrated cerebral electromagnetic field defines two states: the waking state and the state associated with dreaming (rapid eye movement or REM sleep). 
Macro and micro-scale electroencephalographic processes are congruent with the fundamental or first harmonic of the Schumann resonances. A $40 \mathrm{~Hz}$ "ripple" is superimposed upon the classic theta $(4-7 \mathrm{~Hz})$ frequency that is generated by the hippocampal formation [17]. Large portions of input from the cerebral cortices are converged within the entorhinal cortices before being distributed to the hippocampal formation and redistributed to the cerebral cortical manifold [18].

Within layer II of the entorhinal cortices stellate cells exhibit a continuous $\sim 8 \mathrm{~Hz}$ subthreshold oscillation with peak-to-peak values of $2.5 \mathrm{mV}$ [19]. Such direct coupling between theta and gamma activity, which is now evident by Magnetic Resonance Imaging BOLD measurements [20], would allow intercalation of the hippocampus (the "gateway to memory" and the cerebral cortices (the primary locus of "consciousness") such that the process of the representation of experience (memory) and consciousness could be integrated.

At the macro-level, quasi-stable patterns or microstates over the entire cerebral cortices have been reliably mapped. Only about four (4) microstates accommodate about $65 \%$ to 75 $\%$ of the variability in global electroencephalographic potentials [21]. They are remarkably reliable across the person's lifetime. The mean duration of these states when associated with imagery is about $120 \mathrm{~ms}$ or $\sim 8 \mathrm{~Hz}$. Lehmann et al [22] had suggest these four states could be the "atoms of thought" analogous to the base pairs of DNA. If this were valid than $4^{8}$ ( 8 per second) or $6.55 \cdot 10^{4}$ combinations of these microstates would occur per second. This would be equivalent to the information within a low resolution photograph [23].

\section{CONVERGENT TIMES FOR REPRESENTATION}

The time required for the representation of the labile (electrical) component of experience into synaptic patterns, through the emergence of spines on dendrites, is between 15 and $30 \mathrm{~min}$ [24]. This is also the approximate time for micromorphological changes to occur within the region of the hippocampus (CA3) that receives input from the entorhinal cortices. Consequently the electrical patterns must be maintained for this duration, about 1 to $2 \mathrm{ksec}$, in order for the process to be transformed to structural equivalents. This duration has been assumed to be related to the physicochemical restraints for protein synthesis. However it may also involve the latency required to represent information within a component of the space within the resonance cavity [25].

The classic current decay within the electric field between the earth's surface and ionosphere can be obtained by dividing the permittivity of space $\varepsilon_{0}=8.85 \cdot 10^{-12} \mathrm{~F} \cdot \mathrm{m}^{-1}$ by the average conductivity of about $2 \cdot 10^{-14} \mathrm{~S} \cdot \mathrm{m}^{-1}$ [26]. The solution is about $7 \mathrm{~min}$. If about 3 times this base value is required, which is typical for the inflection point of asymptote for many electronic systems, the value would be $\sim 20 \mathrm{~min}$. Similarly the total flux of the earth's magnetic field from its dipole moment $\left(8 \cdot 10^{22} \mathrm{~A} \cdot \mathrm{m}^{2}\right)$ divided by the surface area $\left(5.1 \cdot 10^{14} \mathrm{~m}^{2}\right)$ is $1.6 \cdot 10^{8} \mathrm{~A}$. The inductance, which is flux density $\left(2.55 \cdot 10^{10}\right.$ Webers $)$ divided by amps, would be $1.6 \cdot 10^{2}$ Henrys. The multiplication of this value and a general earth-ionosphere capacitance of 2 Farads [27] and the Schumann resonance of $7.5 \mathrm{~Hz}$ produce a duration of about $2 \mathrm{ksec}$ or $30 \mathrm{~min}$.

These two solutions could reveal a recondite coupling between the Schumann frequencies at intensities almost ten million times less than the static geomagnetic field and this steady volume of potential magnetic energy. The product of the dipole magnetic moment of the earth's field $\left(8 \cdot 10^{22} \mathrm{~A} \cdot \mathrm{m}^{2}\right)$ and the average intensity $\left(5 \cdot 10^{-5} \mathrm{~T}\right)$ is $4 \cdot 10^{18} \mathrm{~J}$. In comparison, if the average human cerebral cortices' $\sim 20$ billion neurons generate $2 \cdot 10^{-20} \mathrm{~J}$ 
quantum of energy with each action potential (neuronal discharge) 10 times per second for the person's life time of two or three Gsec, the total energy associated with experience and thinking would be in the order of 10 to $20 \mathrm{~J}$. Stated alternatively, the earth's magnetic field if coupled to the Schumann resonances would have the potential to represent ("store") the information or neuronal firing patterns from every brain of every human being who has lived on this planet.

\section{POTENTIAL ASTRONOMICAL-COSMOLOGICAL CONNECTIONS}

The potential astronomical relevance of this summed, cerebral quantum from the human species is evident when the energy per $\mathrm{s}$ if transformed to power density of irradiance. Assuming $4 \cdot 10^{-9} \mathrm{~J} \cdot \mathrm{s}^{-1}$ per human brain associated with cerebral activity and $7 \cdot 10^{9}$ human beings on the earth's surface, there would be $\sim 28 \mathrm{~W}$ of power associated with cerebral processes. The earth's surface is $\sim 5 \cdot 10^{14} \mathrm{~m}^{2}$. This means that the power density for this index of cerebral activity for the species would be $\sim 0.6 \cdot 10^{-13} \mathrm{~W} \cdot \mathrm{m}^{-2}$ which is the approximate range of cosmic ray intensity over the earth's surface [3]. Often, but not always, systems that operate within similar narrow limits or corridors of intensity have the potential to intercalate and to exchange information.

Although the primary mechanisms involved with Schumann resonances have focused upon the electric and magnetic components of the propagating waves, the sources of these energies, lightning, is also associated with substantial local emission of photons. The presence of photons in physical and chemical processes increases the probability that excess correlations or entanglements will occur [28]. The convergence of the time required for a photon moving at $\mathrm{c}$ to traverse a plasma cell membrane with a width of $10^{-8} \mathrm{~m}$ is about $10^{-16} \mathrm{~s}$ which is remarkably similar to the duration required for one rotation of an electron around its orbit.

The time required for a singular orbit of an electron, one of the components from which Planck's constant can be derived [29], is in the same order of magnitude. This allows the condition for the information from one unit orbit to be represented during the transmembrane passage of a photon from anywhere and for the information within the membrane to be represented within a photon leaving membrane space.

Spectral analyses of QEEG data simultaneously with biophoton emissions while subjects sat within the dark and imagined light [29] showed maxima in power within the parahippocampal region of the right hemisphere. Both shared phase modulations of about 20 ms. Beat frequencies, which approached the fundamental of the Schumann resonance between peak power in photon emissions and cerebral frequencies, were equivalent to the small, discrete energies differences within the visible wavelength. The right parahippocampal region, in particular, is activated during dream states. The precision of the frequency may be critical. For example Harmony [30] reported QEEG power differences during cognitive processing for children and adults at $7.8 \mathrm{~Hz}$ over the frontal lobes. Power decreased in adults while it increased in children.

The significance of a cosmological interconnection is enhanced when the calculations by Minakov et al [31] are applied. They showed, mathematically, that the gravitational-toelectromagnetic wave conversion in the electrostatic field of the earth converges within the ionosphere resonator. The most power amplification of a plane gravitational wave interacting with a static field inside this natural resonator occurred near the second harmonic of the Schumann resonance, i.e., $14 \mathrm{~Hz}$. Within prominent QEEG profiles and frequencies, this 
second harmonic is the center of the narrow range of 13 to $15 \mathrm{~Hz}$ which defines the symmetrical, classic intermittent "sleep spindles" which occur as bursts with durations of $\sim 1 \mathrm{~s}$ during Stage II sleep.

The $3 \mu \mathrm{V}$ range for the averaged potential of the global QEEG activity is also within the precise range that could be reflected in one of the most prominent frequencies in astrophysics: the neutral hydrogen line. The influence of an averaged $3 \cdot 10^{-6} \mathrm{~V}$ shift upon a unit charge $1.6 \cdot 10^{-19} \mathrm{~A} \cdot \mathrm{s}$ is $4.8 \cdot 10^{-25} \mathrm{~J}$. When divided by the defining constant of quantum phenomena, Planck's constant $\left(6.626 \cdot 10^{-34} \mathrm{~J} \cdot \mathrm{s}\right)$, the equivalent frequency is $0.72 \cdot 10^{9} \mathrm{~Hz}$, which would exactly $1 / 2$ of the $1.42 \mathrm{GHz}$ peaks that defines this line across neutral hydrogen across the universe. Although this coincidence does not prove that the information within the global electric field of cerebral activity would intercalate with the presumably integrated field of neutral hydrogen frequencies within the universe, the congruence might be considered sufficient for further investigation.

\section{MEDIATING CEREBRAL PHYSIOCHEMICAL PROCESSES}

The product of $\mathrm{A} \cdot \mathrm{m}^{-1}$ and $\mu$ is magnetic field intensity (in Tesla); hence, the former can be estimated by calculation. If the cerebral cortical magnetic field strength of $2 \mathrm{pT}$ associated with the QEEG average of $3 \mu \mathrm{V} \cdot \mathrm{Hz}^{-1}$ is divided by magnetic permeability $\left(4 \pi \cdot 10^{-7} \mathrm{~N} \cdot \mathrm{A}^{-2}\right)$, the current would be $1.6 \cdot 10^{-6} \mathrm{~A} \cdot \mathrm{m}^{-1}$. From the average rostral-caudal extent of the cerebrum (17 $\mathrm{cm})$, the net current would be $\sim 2.7 \cdot 10^{-7} \mathrm{~A}$ or the equivalent of about $1.7 \cdot 10^{8}$ charges $(\mathrm{A} \cdot \mathrm{s})$.

The paucity of the charges is revealing. Pakkenberg and Gundersen [32] estimated an average total of $\sim 23$ and $\sim 19$ billions of neurons within the neocortices of the average man and woman respectively, where $\mathrm{CV}$, the coefficient of variation was 0.17 for both. Because the action potential is associated with the movement of $\sim 2 \cdot 10^{7}$ charges or ions, a total of 3.2 to $4 \cdot 10^{17}$ charges would theoretically be involved per s. This discrepancy between $10^{8}$ and $10^{17}$ charges would be accommodated if the source of this charge was the "free" protons $\left(\mathrm{H}^{+}\right)$ associated with the $\mathrm{H}_{3} \mathrm{O}^{+}$(hydronium ion). According to DeCoursey [33] "only one proton in a billion is a part of the hydronium ion at any moment".

\section{CONCLUSIONS}

The evolution of the physical chemical and biological phenomena that have aggregated to produce complex organisms and the electromagnetic processes that emerged within their spatial boundaries has occurred while immersed in the geomagnetic field and the intensities and frequencies generated as Schumann resonances within the earth-ionosphere cavity. The remarkable convergences between the temporal patterns and the intensities of the magnetic and electric field components for Schumann resonances found within both global human quantitative electroencephalographic activity and earth-ionosphere activity could suggest a causal relationship. Whether or not a type of conscious or cognition-like phenomena occurs within the global state of the earth-ionosphere to which all human beings are normally exposed may not be as important as the multiplicity of subtle influence upon the properties of the Schumann resonances from astronomical phenomena associated with solar activity, gravitational waves, and cosmic ray modulations. 


\section{References}

[1] A. P. Johnson, J.J. Cleaves, J. P. Dworkin, D. P. Glavin, A. Lazcano, J. L. Bada, Science $322(2008) 404$.

[2] M. A. Persinger (ed), ELF and VLF Electromagnetic Field Effects. Plenum Press, New York, 1974, pp.

[3] H. L. Koenig, A. P. Krueger, S. Lang, W. Sonnig, Biologic Effects of Environmental Electromagnetism. Spring-Verlag, NewYork, 1981.

[4] N. Cherry, Natural Hazards 26 (2002) 279-331.

[5] A. Nickolaeno, M. Hayakawa Schumann Resonances for Tyros. Springer, Tokyo, 2014.

[6] S. Matsushita, W. H. Campbell, Physics of Geomagnetic Phenomena. Academic Press, New York, 1967.

[7] K. Schlegel, M. Fullekrug, Journal of Geophysical Research 104 (1999) 10,111-10,118.

[8] C-y. T. Li, M-m. Poo, Y. Dan, Science 324 (2009) 643-645.

[9] D. Kahn, E. F. Pace-Schott, J. A. Hobson, Neuroscience 78 (1997) 13-38.

[10] B. Alberts, A. Johnson, J. Lewis, M. Raff, K. Roberts, P. Walter, Molecular Biology of the Cell. Garland Science, New York, 2002.

[11] M. A. Persinger, S. A. Koren, G. F. Lafreniere, NeuroQuantology 6 (2008) 262-271.

[12] A. Delorme, S. Makeig, Journal of Neuroscience Methods 134 (2004) 9-21.

[13] G. Ryskin, New Journal of Physics 11 (1995) 063015.

[14] J. P. Wikswo, J. P. Barach, J. A. Freeman, Science 208 (1980) 53-55.

[15] P. L. Nunenz, Neocortical Dynamics and Human EEG Rhythms. Oxford University Press, New York, 1995.

[16] R. R. Llinas, D. Pare, Neuroscience 44 (1991) 521-535.

[17] G. Buzaski, Neuron 33 (2002) 325-340.

[18] P. Gloor, The Temporal Lobe and Limbic System (1997) Oxford University Press: N.Y.

[19] A. Alonso, R. Klink, Journal of Neurophysiology 70 (1993) 128-143.

[20] J. C. Whitman, L. M. Ward, T. Woodward, Frontiers in Human Neuroscience 7 (2013) Article 80.

[21] T. Koenig, L. Prichep, L. Lehmann, D. V. Sosa, E. Braker, H. Kleinlogel, R.Ishehart, E. R. John, NeuroImage 16 (2002) 41-48.

[22] D. Lehmann, W. K. Strik, B. Henggeler, T. Koenig, M. Koukkou, International Journal of Psychophysiology 29 (1998) 1-11.

[23] M. A. Scott, M. A. Persinger, Journal of Signal and Information Processsing 4 (2013) 282-287.

[24] I. Nikonenko, P. Jourdain, S. Alberi, N. Toni, D. Muller, Hippocampus 12 (2002) 585-591.

[25] M. A. Persinger, Theoretical Biology Insights 1 (2008) 3-11. 
[26] H. Voland, Handbook of Atmospherics Volume I. CRC Press, Boca Raton (Fla), 1982, p. 66.

[27] R. Hill, Pure and Applied Geophysics 84 (1971) 67-74.

[28] B. T. Dotta, M. A. Persinger, Journal of Biological Chemistry 3 (2012) 72-80.

[29] B. T. Dotta, K. S. Saroka, M. A. Persinger, Neuroscience Letters 513 (2012) 151-154.

[30] T. Harmony, Frontiers in Integrative Neuroscience 7 (2013) Article 83, 1-10.

[31] A. A. Minakov, A. P. Nikolaenko, L. M. Rabinovich, Radiofizika 35 (1992) 488-497.

[32] B. Pakkenberg, J. G. Gundersen, The Journal of Comparative Neurology 384 (1997) 312-320.

[33] T. E. DeCoursey, Physiological Review 83 (2002) 475-579. 